\title{
Clinical and virological analysis of epidemic keratoconjunctivitis caused by adenovirus type 54 in a regional ophthalmic clinic in Kyushu, Japan
}

This article was published in the following Dove Press journal:

Clinical Ophthalmology

\author{
Takashi Uemura' \\ Hironori Migita ${ }^{2}$ \\ Tomohiro Ueno \\ Tomoko Tsukahara- \\ Kawamura ${ }^{1,3}$ \\ Yusuke Saeki' \\ Tsuguto Fujimoto 3 \\ Eiichi Uchio' \\ 'Department of Ophthalmology, \\ Fukuoka University School of \\ Medicine, Fukuoka, Japan; ${ }^{2}$ Migita Eye \\ Clinic, Chikushino, Japan; ${ }^{3}$ Infectious \\ Disease Surveillance Center, National \\ Institute of Infectious Diseases, \\ Tokyo, Japan
}

Background: Human adenovirus type 54 (HAdV-54) is a novel type of adenovirus that belongs to species D and has thus far been detected only in Japan in patients with epidemic keratoconjunctivitis (EKC). There was a large nationwide outbreak of HAdV-54 EKC from 2015 to 2016 in Japan. The clinical characteristics of an outbreak of HAdV-54 conjunctivitis treated in a regional ophthalmic clinic in Fukuoka, Japan, in 2016 were analyzed.

Patients and methods: A consecutive series of 55 cases diagnosed clinically as EKC confirmed by HAdV-54 detection from conjunctival scrapings by polymerase chain reaction (PCR) method between 17 June 2016 and 29 August 2016 were enrolled. Viral DNA copies were counted by realtime PCR method. The clinical findings were recorded at the first visit to the clinic and evaluated. Results: In the analysis of the relationship between mean clinical score groups and several factors, such as days after onset, sex, HAdV DNA number on a logarithmic scale, and age, most factors did not show a significant difference in clinical score between groups. However, mean clinical score of cases aged $<23$ years was significantly higher than that of cases aged $\geq 23$ years $(P<0.01)$. The correlation coefficient between DNA copies on a logarithmic scale and clinical score was 0.280 , and a significant correlation was observed $(P<0.05)$. Multiple subepithelial corneal infiltrates (MSI) were observed in 24 out of 31 cases $(77 \%)$.

Conclusion: These results suggested that the clinical features in the early phase of HAdV-54 keratoconjunctivitis were milder but the rate of MSI observed in the late phase was higher than those in previous epidemics of several HAdV types. The significant difference in clinical severity between age groups might be a virological characteristic of HAdV-54.

Keywords: adenovirus, epidemic keratoconjunctivitis, type 54, multiple subepithelial infiltrates

\section{Introduction}

Epidemic keratoconjunctivitis (EKC) is an acute viral disease of the eye which is caused by several adenovirus types, such as type 8,37 , and $64 .{ }^{1}$ Outbreaks caused by several adenovirus types have been reported in various eye-care institutions in several countries. $^{2-6}$ Human adenovirus type $54(\mathrm{HAdV}-54)$ is a novel type of adenovirus ${ }^{7}$ that belongs to species D which causes EKC. ${ }^{8-10}$ Until recently, it had been detected only in Japan, but it was reported to have been detected in Greece lately. ${ }^{7} \mathrm{HAdV}-54$ was identified as a HAdV-8 variant strain before 2008, because antibodies to HAdV-54 showed cross-reactivity with those to $\mathrm{HAdV}-8$ in a neutralization test. ${ }^{8,10}$ Virological aspects of HAdV-54 have been reported in the above studies using phylogenetic analysis; however, in contrast, the clinical features of acute conjunctivitis due to HAdV-54 have been reported in a recent study by Akiyoshi et al. ${ }^{11}$ They reported an outbreak of EKC in a nursery school and stated that the clinical presentation in the
Correspondence: Eiichi Uchio Department of Ophthalmology, Fukuoka University School of Medicine, 7-45-I Nanakuma, Jonan-ku,

Fukuoka 8I4-0180, Japan

Tel +81928011011

Fax +8I 928654445

Email euchio@fukuoka-u.ac.jp 
early stage of HAdV-54 infection resembled that of acute allergic conjunctivitis and then subsequently described the typical features of severe EKC including severe corneal opacity especially in adult cases treated with corticosteroid eye drops. ${ }^{11}$ However, the study population of this study in which HAdV-54 was detected by polymerase chain reaction (PCR) method was $<10$ and information from clinical analysis was limited. There was a large nationwide outbreak of HAdV-54 EKC from 2015 to 2016 in Japan. ${ }^{12}$ However, there has been no study on the genomic evolution among HAdV-54 from recent strains which might be the reason for the increasing epidemics of EKC caused by HAdV-54. In this report, we analyzed the clinical characteristics of an outbreak of HAdV-54 conjunctivitis treated in a regional ophthalmic clinic in Fukuoka, Japan in 2016.

\section{Patients and methods Study populations}

In June 2016, a private ophthalmology clinic in Chikushino, Fukuoka, Japan encountered an epidemic of EKC which was prolonged for 3 months. The clinic was one of the ophthalmology practices in the region and served a population of about 20,000 people. Chikushino is located in the suburban area of Fukuoka, which has a population of 1,550,000 people and is the largest city in southwestern Japan. This was a consecutive case series study of 55 cases (23 men and 32 women) who attended Migita Eye Clinic between 17 June 2016 and 29 August 2016 and were diagnosed clinically as having EKC according to the typical clinical findings, such as acute unilateral or bilateral viral conjunctivitis (with characteristic clinical features, such as sudden onset of acute follicular conjunctivitis with watery discharge, hyperemia and chemosis, and preauricular lymphadenopathy). The diagnosis was confirmed by HAdV-54 detection from conjunctival scrapings by the method reported previously using PCR as described later. ${ }^{13}$ To prevent nosocomial outpatient infection, frequent hand washing by physicians and disinfection of clinical apparatus was performed, and all cases were advised of standard precautions to avoid intrafamilial infection. Immunochromatography test for HAdV was also carried out in all cases. Exclusion criteria included a history of seasonal allergic conjunctivitis, contact lens use, a history of herpetic eye disease, and a history of ocular surgery. Cases that showed a negative result of PCR for HAdV or a positive result of PCR for other types than HAdV-54 were also excluded. Written informed consent for the use of the clinical samples was obtained from all patients included in this study. This study was approved by the Ethics Review Committee of Fukuoka University (16-2-15).

\section{Clinical observation and grading}

Ocular findings of slit lamp examination were recorded on the patients' first visit. Two objective ocular clinical findings of corneal and conjunctival lesions were graded on a 4 -point scale $(0=$ none, $1=$ mild, $2=$ moderate, $3=$ severe; left and right eye separately in each case). Corneal findings were graded as follows: mild $=$ superficial punctate keratitis $(\mathrm{SPK})$, moderate $=$ corneal erosion, and severe $=$ corneal ulcer. Conjunctival lesions were graded according to the criteria reported by Aoki et al ${ }^{14}$ with modification as follows: mild = inflammation confined to the lower palpebral conjunctiva, moderate $=$ inflammation extending to the upper palpebral conjunctiva but not affecting the fornix, and severe $=$ inflammation of the fornix of the upper palpebral conjunctiva and/or pseudomembrane formation. Preauricular lymphadenopathy was graded as 0 (absent) or 1 (present) on each side. These three scores were summed and used as the clinical score of the eye. In bilateral cases, the score of the more severe eye, mostly the firstly infected eye, was used as the clinical score of the case. Multiple subepithelial corneal infiltrates (MSI) were recorded during the follow-up period. Therefore, if the patient did not attend after the acute phase, MSI could not be observed in that case.

\section{Virological analysis}

Conjunctival scrapings of the affected eyes were tested by an immunochromatography test (Quick Nave Adeno; Denka Seiken Co. Ltd., Tokyo, Japan) at the patients' first visit. Regardless of the result of immunochromatography test, the remaining conjunctival swab samples were frozen and stored at $-30^{\circ} \mathrm{C}$ until they were used for quantitative PCR amplification. Sequencing and phylogenetic analysis to detect the presence of HAdV and typing were carried out on all stored samples on December 2016 as follows. DNA was extracted from these specimens using a QIA $\operatorname{amp}^{\circledR}$ DNA mini kit (Qiagen NV, Venlo, the Netherlands), and the copy number was measured with real-time PCR based on the primers and conditions in a previous report. ${ }^{15}$ Forward and reverse primers were GACATGACTTTCGAGGTCGATCCCATGGA and CCGGCTGAGAAGGGTGTGCGCAGGTA, respectively. ${ }^{15}$ Forty cycles of PCR were performed, and each cycle consisted of the following incubations: $95^{\circ} \mathrm{C}$ for 10 seconds and $56^{\circ} \mathrm{C}$ for 30 seconds. Sequencing for typing was carried out according to a previous report. ${ }^{13}$ Primers were DGF (forward); AGACATGACHTTYGAGGTGGAYCC and DGR (reverse); CGCAGGTASACBGYYTCRA, and a probe targeted for species D HAdV was 98D; TCAGAGTGCAC CAGCCGCACC. ${ }^{13}$ Fifty cycles of PCR were performed, 
and each cycle consisted of the following incubations: $95^{\circ} \mathrm{C}$ for 5 seconds and $60^{\circ} \mathrm{C}$ for 30 seconds. The sequences were analyzed using a sequencer (Gene Codes Corporation, Ann Arbor, MI, USA) and BioEdit Version 7.2.1 (Ibis Therapeutics, Carlsbad, CA, USA). Phylogenetic analyses were performed and trees were generated using MEGA5 (CEMI, Tempe, AZ, USA).

\section{Statistical analysis}

SPSS 19.0 software was used for data analysis (IBM, Armonk, NY, USA). Since it has not been established whether the clinical score of viral conjunctivitis shows a normal distribution, non-parametric analysis was conducted in this study. Mann-Whitney $U$ test was used to identify differences in mean values among patient groups. Spearman's rank correlation test was used to establish the significance of correlation between paired groups. A value of $P<0.05$ was accepted as statistically significant.

\section{Results}

Demographic profiles of the cases in this study are shown in Table 1. Mean age was 21.7 years because a considerable proportion of cases (32 cases; $58.2 \%$ ) were children (below 16 years of age). Most cases attended Migita eye clinic within 3 days after the onset; however, six cases (11\%) attended $\geq 4$ days after the onset.

Mean clinical score of cases who attended the clinic within 2 days after the onset did not show a significant difference from those who attended $\geq 3$ days after the onset. No significant difference in mean clinical score was observed between men and women. When the patients were divided into two groups according to the results of real-time PCR of HAdV DNA number on a logarithmic scale, there was no significant difference in mean clinical score between the $\geq 8.6$ group and $<8.6$ group (Table 2). However, the mean clinical score of cases aged $<23$ years $(2.69 \pm 0.53)$ was significantly higher than that of cases aged $\geq 23$ years (2.22 $\pm 0.51 ; P<0.01$; Table 2).

Table I Demographic features of cases of epidemic keratoconjunctivitis due to adenovirus type 54

\begin{tabular}{lll}
\hline & & Range \\
\hline Sex & & \\
Men & $23(42 \%)$ & \\
Women & $32(58 \%)$ & \\
Age (mean \pm SD) & $21.7 \pm 22.7$ & $0-68$ \\
Day of first visit after onset (mean \pm SD) & $1.69 \pm 1.52$ & $0-6$ \\
No. of HAdV copies (log copies/mL; mean \pm SEM) & $8.50 \pm 0.93$ & $5.0-10.1$ \\
\hline Abbreviations: HAdV, human adenovirus; SD, standard deviation; SEM, standard &
\end{tabular}

Table 2 Relationship between clinical score and patient groups

\begin{tabular}{lll}
\hline $\begin{array}{l}\text { Classification } \\
\text { (number of cases) }\end{array}$ & $\begin{array}{l}\text { Clinical score } \\
\text { (mean } \pm \text { SD) }\end{array}$ & $\begin{array}{l}\text { Statistical } \\
\text { significance }\end{array}$ \\
\hline $\begin{array}{l}\text { Days after onset } \\
\leq 2(42)\end{array}$ & NS \\
$3 \leq(13)$ & $2.52 \pm 0.59$ & \\
Sex & $2.38 \pm 0.49$ & NS \\
$\quad$ Men (23) & $2.61 \pm 2.4 \mathrm{I}$ & \\
Women (32) & $28.9 \pm 0.6 \mathrm{I}$ & \\
HAdV DNA number on logarithmic scale & NS \\
$\leq 8.6(29)$ & $2.4 I \pm 0.56$ & \\
$>8.6(26)$ & $2.58 \pm 0.57$ & $P<0.0 \mathrm{I}$ \\
Age (years) & & \\
$<23(32)$ & $2.69 \pm 0.53$ & \\
$\geq 23(23)$ & $2.22 \pm 0.5 \mathrm{I}$ &
\end{tabular}

Abbreviations: HAdV, human adenovirus; NS, not significant.

The correlation between DNA copies on a logarithmic scale and day of sample collection after the onset was analyzed; the correlation coefficient was -0.237 , but no significant correlation was observed (Figure 1). In contrast, the correlation coefficient between DNA copies on a logarithmic scale and clinical score was 0.280 , and a significant correlation was observed $(P<0.05$; Figure 2$)$.

The clinical severity of conjunctivitis was mild in $3.6 \%$, moderate in $94.6 \%$, and severe in $1.8 \%$. All cases showed moderate grade conjunctivitis (Figure 3) except for three cases. No case exhibited a conjunctival pseudomembrane. Preauricular lymphadenopathy was observed in 27 cases (49\%). In contrast, corneal involvement was found in only one case as SPK (1.8\%), and corneal erosion or ulcer was not observed. Bilateral involvement was present in $14.5 \%$ (eight cases). MSI were observed in 24 out of 31 cases (77\%) that were followed up several times after the acute phase because of the need for extended topical treatment. Serious systemic

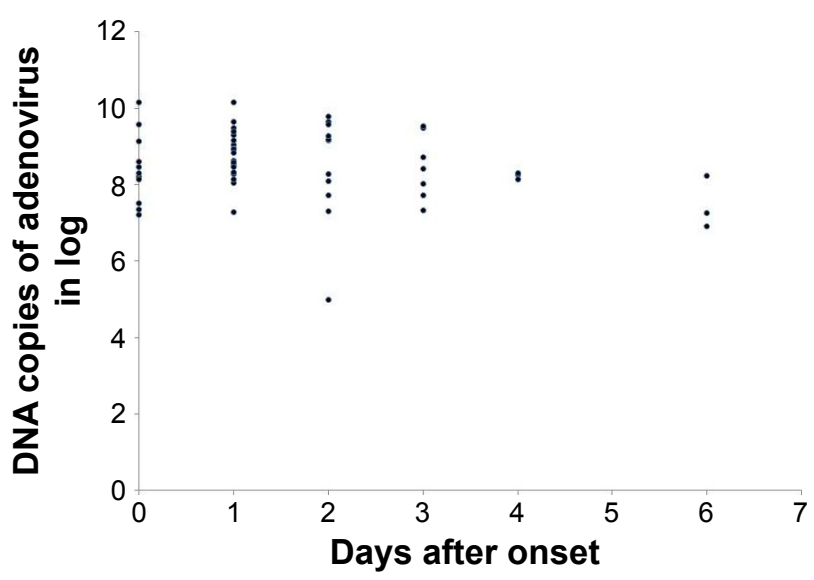

Figure I Correlation between AdV DNA copies on logarithmic scale and day of sample collection after onset. Correlation coefficient was -0.237 , but this correlation was not significant. DNA copies of adenovirus in log are represented in $Y$-axis. Abbreviation: $\mathrm{AdV}$, adenovirus. 


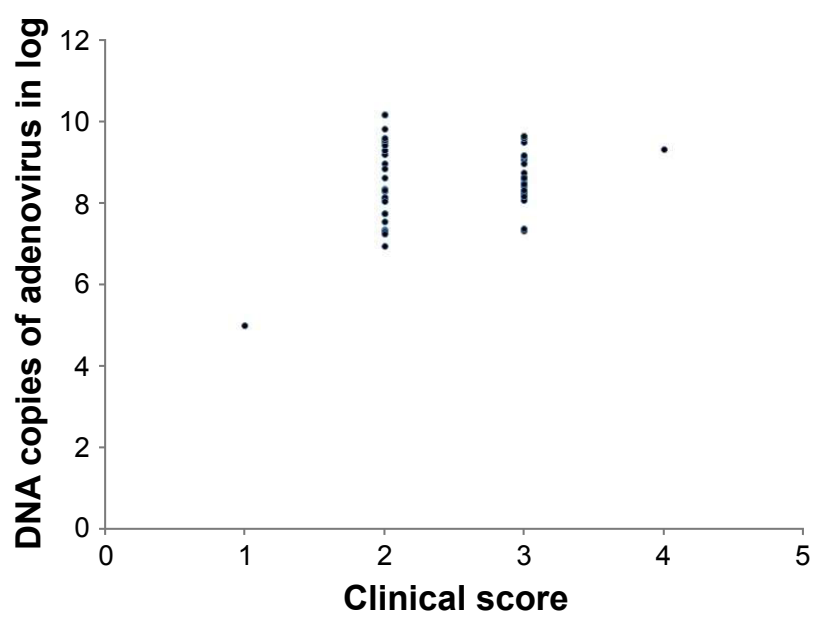

Figure 2 Correlation between AdV DNA copies on logarithmic scale and clinical score. Correlation coefficient was 0.280 and a significant correlation was observed $(P<0.05)$. DNA copies of adenovirus in log are represented in $Y$-axis.

Abbreviation: AdV, adenovirus.

complicating infection such as pneumonia or hemorrhagic cystitis was not observed among the study population.

\section{Discussion}

The complete genomic sequence of HAdV-54 (Kobe-H strain) was deposited in GenBank (accession no AB333801) as the prototype strain. ${ }^{8}$ As mentioned earlier, HAdV-54 has caused epidemics continuously only in Japan in the past decade. ${ }^{11}$ However, there have been few studies describing its clinical features. It has been reported that a high incidence of MSI (53\%) was observed in an epidemic of EKC due to HAdV-54 occurring in a nursery school in Kobe City, Japan in $2008 .^{11}$ However, the detailed features of the clinical findings of EKC due to HAdV-54 have not been previously reported. There was a difference in the study population between the present study and that of Akiyoshi et al. ${ }^{11}$ We reported a regional community-scale epidemic in a broad age group; in contrast, Akiyoshi et al reported a nosocomial infection

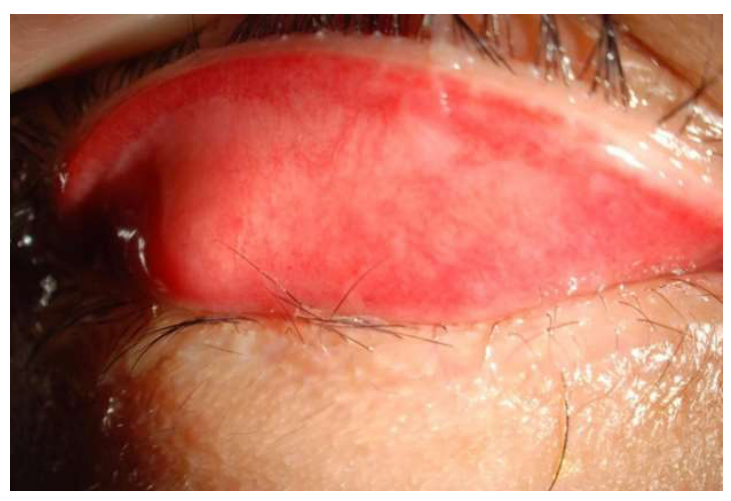

Figure 3 Conjunctival finding of case of AdV type 54. Moderate follicular conjunctivitis with eye discharge of a 32-year-old man is shown.

Abbreviation: AdV, adenovirus. in nursery school children and their parents. A similarity of these studies is that both epidemics were prolonged for over 3 months in the summer season.

There have been several studies reporting the clinical characteristics of adenoviral keratoconjunctivitis as shown in Table 3. Tullo and Higgins reported higher incidences of conjunctival pseudomembrane (37\%) and corneal involvement including dendritic keratitis (68\%) in an outbreak caused by HAdV-10 and $-19 .{ }^{16}$ The HAdV-19 strain that causes acute follicular conjunctivitis is currently named HAdV-64. ${ }^{17}$ The rate of keratitis was reported to be $9.1 \%, 27 \%, 61 \%$, and $70 \%$ with HAdV-3, $-4,-8$, and -19 , respectively. ${ }^{18}$ The incidence of preauricular lymphadenopathy ranged from $20 \%$ to $64 \%$, and the highest rate was observed in HAdV-19-induced keratitis. ${ }^{18}$ Darougar et al also reported the clinical characteristics of adenoviral keratoconjunctivitis in eight types from 98 consecutive patients. ${ }^{19}$ Both SPK and MSI were observed in $95 \%$ of HAdV-8 EKC cases, and a conjunctival pseudomembrane was found in $38 \% .{ }^{19}$ In contrast, the rate of pseudomembrane in species B types (HAdV-3 and -7) was lower (0\%-17\%), and the rates of SPK and MSI were also lower $(56 \%-83 \%$ and $17 \%-25 \%$, respectively) compared with HAdV-8. ${ }^{19}$ Aoki et al recently reported that they observed preauricular lymphadenopathy and MSI in $23.5 \%$ and $43 \%$ of 68 cases, respectively, in adenoviral keratoconjunctivitis due to several types. ${ }^{14}$ Huang et al reported a recent outbreak of EKC caused by HAdV-56, which belongs to species D, in two factories in China in which acute conjunctivitis was observed in $91 \%$ of the cases; however, corneal complications were not mentioned. ${ }^{20}$

Regarding the clinical severity of conjunctivitis, most cases $(94.6 \%)$ were moderate grade in our study. The reason for this high uniformity of clinical severity was presumed to be due to the short-length single epidemic in this study. Compared with past reports of conjunctival findings, the overall severity of conjunctivitis in which $96 \%$ of cases showed moderate conjunctivitis was milder than that reported by Aoki et al who stated that more than $40 \%$ of cases with HAdV-8 or HAdV-19 showed severe conjunctivitis. ${ }^{18}$ Although the recent study reported by Aoki et al $^{16}$ included several types, the major proportion of cases was induced by species D (87\%) HAdV and HAdV-54 comprised 19\%, and the distribution of severity of conjunctivitis was similar to our results. Regarding conjunctival pseudomembrane, the reported incidence ranged from $37 \%$ to $38 \%$ in the studies by Tullo and Higgins (HAdV-10 and -19) ${ }^{16}$ and Darougar et al (HAdV-8). ${ }^{19}$ In contrast, no case showed pseudomembrane in our study. Combining these results, $\mathrm{HAdV}-54$-induced conjunctivitis 
Table 3 Reports of clinical characteristics of adenoviral keratoconjunctivitis in past studies and present study

\begin{tabular}{|c|c|c|c|}
\hline Clinical findings & Incidence (\%) & Study & $\begin{array}{l}\text { Adenovirus } \\
\text { species and type }\end{array}$ \\
\hline Conjunctival & 37 & Tullo and Higgins ${ }^{16}$ & DIO and DI9* \\
\hline \multirow[t]{3}{*}{ pseudomembrane } & 17 & Darougar et $\mathrm{al}^{19}$ & B7 \\
\hline & 38 & & D8 \\
\hline & 0 & Uemura (present study) & D54 \\
\hline \multirow[t]{13}{*}{ Keratitis } & 68 & Tullo and Higgins ${ }^{16}$ & DIO and DI9* \\
\hline & 56 & Aoki et al ${ }^{18}$ & B3 \\
\hline & 9.1 & & $\mathrm{E} 4$ \\
\hline & 27 & & D8 \\
\hline & 61 & & DI9* \\
\hline & 70 & Darougar et al ${ }^{19}$ & B3 \\
\hline & 75 & & E4 \\
\hline & 100 & & $\mathrm{C5}$ \\
\hline & 83 & & B7 \\
\hline & 95 & & D8 \\
\hline & 100 & & BII \\
\hline & 50 & & DI5 and DI5/29 \\
\hline & 1.8 & Uemura (present study) & D54 \\
\hline Preauricular & 37 & Tullo and Higgins ${ }^{16}$ & DIO and DI9* \\
\hline \multirow[t]{7}{*}{ lymphadenopathy } & 20 & Aoki et al ${ }^{18}$ & B3 \\
\hline & 34 & & E4 \\
\hline & 44 & & D8 \\
\hline & 64 & & DI9* \\
\hline & 24 & Aoki et $\mathrm{al}^{14}$ & B3, E4, DI9a", \\
\hline & & & D53 and D54 \\
\hline & 49 & Uemura (present study) & D54 \\
\hline Multiple subepithelial & 58 & Tullo and Higgins ${ }^{16}$ & DIO and DI9* \\
\hline \multirow[t]{9}{*}{ corneal infiltrates } & 25 & Darougar et al ${ }^{19}$ & B3 \\
\hline & 25 & & E4 \\
\hline & 100 & & $\mathrm{C5}$ \\
\hline & 17 & & B7 \\
\hline & 95 & & D8 \\
\hline & 53 & Akiyoshi et al" & D54 \\
\hline & 43 & Aoki et $\mathrm{al}^{14}$ & B3, E4, DI9a", \\
\hline & & & D53 and D54 \\
\hline & 77 & Uemura (present study) & D54 \\
\hline
\end{tabular}

Notes: HAdV-19 (DI9)* has been renamed as HAdV-64 (D64). This is almost identical to HAdV-DI9a", which is a genotype of HAdV-19 causing keratoconjunctivitis.

seems clinically milder than that with other HAdV species D, such as HAdV-8 or HAdV-64. A similar tendency was also noted in the severity of corneal involvement in the acute phase. Keratitis was observed in $1.8 \%$ of cases in our study, whereas the incidence of keratitis ranged from $9.1 \%$ to $95 \%$ in previous studies of adenoviral keratoconjunctivitis. ${ }^{16,18,19}$ This low rate was similar to that in species B adenoviral conjunctivitis, $0 \%-17 \%$ for HAdV-3 and -7 , respectively, reported by Darougar et al. ${ }^{19}$ Compared with the past incidence of preauricular lymphadenopathy ranging from $20 \%$ to $64 \%,{ }^{14,16,18}$ the rate in our study in HAdV-54 cases (49\%) was the highest among HAdV types except for HAdV-19 $(64 \%),{ }^{18}$ indicating that different clinical characteristics were observed in our study for conjunctival or corneal involvement and preauricular lymphadenopathy. The incidence of MSI was $77 \%$ in our study, which was the highest among previous studies, ${ }^{11,14,16,19}$ except for $95 \%$ reported by Darougar et al in cases with HAdV-8. ${ }^{19}$ This incidence was higher than that in the epidemic of HAdV-54 (53\%). ${ }^{11}$ The reason for this discrepancy in the incidence of MSI in the same HAdV-54 conjunctivitis is unclear, but it might have resulted from the difference in age distribution, with more adult cases included in our study than in that of Akiyoshi et al. ${ }^{11}$ However, there remains a possibility that considerable viral evolution might have occurred in the interval between the epidemics, in 2008 and 2016. Thus, complete genomic sequence evaluation of these HAdV-54 samples will be necessary for further analysis. The pathogenesis of MSI in adenoviral keratoconjunctivitis has not been clarified; however, it has been regarded as an immune reaction because of the effectiveness of topical 
corticosteroid treatment. ${ }^{21}$ These high rates of preauricular lymphadenopathy and MSI suggest that the immunological reaction occurring in the course of HAdV-54 keratoconjunctivitis is relatively more active compared with other HAdV types, even though its clinical features on ocular surface are milder in the early phase.

We analyzed the relationship between mean clinical score and several factors, such as days after onset, sex, HAdV DNA number on a logarithmic scale, and age in this study. No factor showed a significant difference in clinical score between the two groups except for patients' age. It is interesting that the younger group had a significantly more severe score than the older group; however, this aspect of analysis in adenoviral conjunctivitis has not been reported in past publications. Considering the high uniformity of clinical grading of conjunctival lesions in this study, the significant difference might have derived from high clinical scores of corneal findings or preauricular lymphadenopathy. The reason for the result is unclear; however, there are several possible explanations for the age-related characteristic of adenoviral keratoconjunctivitis, especially that due to HAdV-54. A recent study revealed age-related changes in conjunctival structure, ${ }^{22}$ leading to the hypothesis that an immature conjunctival structure might have resulted in more severe conjunctival or corneal lesions in the younger group, especially in the pediatric population, in our study. However, this could not explain the specific pathological mechanism in HAdV-54 keratoconjunctivitis. In juvenile cases, more primary infection is seen than in adult cases, leading to the hypothesis that clinically severe cases are more often observed in this age group. This could be supported by the fact that many nosocomial infections of adenoviral keratoconjunctivitis have been reported in pediatric wards including neonatal intensive care units ${ }^{23-25}$ and nursery schools. ${ }^{11}$ However, it is important that all age groups were enrolled in this study in a regional epidemic of HAdV-54 keratoconjunctivitis; therefore, a clinical difference between age groups was able to be revealed with less bias in this study. Regarding the age distribution of the epidemic, there have been a few studies reporting the mean age of cases on a regional clinic basis. Comparing the mean age of 21.7 years in this study, Huang et al reported a median age of 24 years $^{20}$ and Darougar et al reported that the majority of patients were aged between 20 and 39 years, but half of the patients with HAdV-7 were aged $<20$ years. ${ }^{19}$ These showed a similar tendency to the age distribution in our study.

Another important finding of the present study is that there was no significant difference in clinical severity between the higher virus DNA copy number group and lower virus DNA copy number group obtained by quantitative PCR method (Table 1). However, we found a significant correlation between DNA copies on a logarithmic scale and clinical score (Figure 2). These results seem contradictory. Although nonparametric analysis was used in this study, the ordinal scale characteristic of the clinical score might affect the results of comparisons between groups. There are several additional possible explanations for this discrepancy. Neutralizing antibody, that acts to suppress viral proliferation during the clinical course, is reported to rise around 10 days after the onset in human adenoviral conjunctivitis. ${ }^{26}$ However, it is also reported that a rise in antibody titer is observed in $75 \%$ of patients with adenoviral keratoconjnctivitis, ${ }^{26}$ meaning that some patients are non-responders to neutralizing antibody. Combined with this information, the inhibitory effect of neutralizing antibody is very complicated and variable in adenoviral conjunctivitis. Another aspect is that the host immune status affected clinical findings regardless of adenovirus DNA copies. This was presumed from the atypical clinical appearance in specific immunological situations such as immunocompromised cases..$^{27}$ The wide range of date of sample collection for real time PCR method might also have affected our results. Further study will be needed for more detailed evaluation of the relation between the number of viral DNA copies and the clinical severity of adenoviral keratoconjunctivitis, using a more quantitative method. Since specific anti-adenoviral agents have not been introduced locally or systemically at present, it can be considered that the adenovirus DNA copies in our study were not affected by therapeutic measures.

We found several important findings from a regional epidemic of HAdV-54 keratoconjunctivitis. The clinical features in the early phase of keratoconjunctivitis were milder but the rate of MSI observed in the late phase was higher than those in previous epidemics of several HAdV types including a recent study of HAdV-54 keratoconjunctivitis in Japan. ${ }^{11}$ Clinical severity was significantly higher in the young age group than in the older age group; however, no significant difference was observed between the higher viral DNA copies group and lower group. For more detailed analysis to clarify the reason for these observations, genome-level sequential analysis will be needed.

\section{Acknowledgments}

This work was supported by a Grant-in-Aid for Encouragement of Scientists (15K10911) from the Ministry of Education, Science, Sports, and Culture of Japan. We thank Dr W Gray for editing this manuscript. 


\section{Disclosure}

The authors report no conflicts of interest in this work.

\section{References}

1. Jhanji V, Chan TC, Li EY, Agarwal K, Vajpayee RB. Adenoviral keratoconjunctivitis. Surv Ophthalmol. 2015;60(5):435-443.

2. Montessori V, Scharf S, Holland S, Werker DH, Roberts FJ, Bryce E. Epidemic keratoconjunctivitis outbreak at a tertiary referral eye clinic. Am J Infect Control. 1998;26(4):399-405.

3. Colon L. Keratoconjunctivitis due to adenovirus type 8: report on a large outbreak. Ann Ophthalmol. 1991;23(2):63-65.

4. Gottsch J, Froggatt JW 3rd, Smith DM, et al. Prevention and control of epidemic keratoconjunctivitis in a teaching eye institute. Ophthalmic Epidemiol. 1999;6(1):29-39.

5. Barnard DL, Hart JC, Marmion VJ, Clarke SK. Outbreak in Bristol of conjunctivitis caused by adenovirus type 8 , and its epidemiology and control. BMJ. 1973;2(5859):165-169.

6. D'Angelo L, Hierholzer JC, Holman RC, Smith JD. Epidemic keratoconjunctivitis caused by adenovirus type 8 : epidemiologic and laboratory aspects of a large outbreak. Am J Epidemiol. 1981;113(1):44-49.

7. Balasopoulou A, Kokkinos P, Pagoulatos D, et al. A molecular epidemiological analysis of adenoviruses from excess conjunctivitis cases. BMC Ophthalmol. 2017;17(1):51.

8. Ishiko H, Shimada Y, Konno T, et al. Novel human adenovirus causing nosocomial epidemic keratoconjunctivitis. J Clin Microbiol. 2008; 46(6):2002-2008.

9. Ishiko H, Aoki K. Spread of epidemic keratoconjunctivitis due to a novel serotype of human adenovirus in Japan. J Clin Microbiol. 2009;47(8): 2678-2679.

10. Kaneko H, Suzutani T, Aoki K, et al. Epidemiological and virological features of epidemic keratoconjunctivitis due to new human adenovirus type 54 in Japan. Br J Ophthalmol. 2011;95(1):32-36.

11. Akiyoshi K, Suga T, Fukui K, Taniguchi K, Okabe N, Fujimoto T. Outbreak of epidemic keratoconjunctivitis caused by adenovirus type 54 in a nursery school in Kobe City, Japan in 2008. Jpn J Infect Dis. 2011; 64(4):353-355.

12. Suzuki S, Kawamura T, Saeki Y, et al. A case of type 54 human mastadeno virus keratoconjunctivitis causing severe broad epithelial defect ten years after LASIK surgery. Jpn J Infect Dis. 2017. Epub July 1.

13. Gu Z, Belzer SW, Gibson CS, Bankowski MJ, Hayden RT. Multiplexed, real-time PCR for quantitative detection of human adenovirus. J Clin Microbiol. 2003;41(10):4636-4641.
14. Aoki K, Kaneko H, Kitaichi N, Ohguchi T, Tagawa Y, Ohno S. Clinical features of adenoviral conjunctivitis at the early stage of infection. Jpn J Ophthalmol. 2011;55(1):11-15.

15. Watanabe M, Kohdera U, Kino M, et al. Detection of adenovirus DNA in clinical samples by SYBR Green real-time polymerase chain reaction assay. Pediatr Int. 2005;47(3):286-291.

16. Tullo AB, Higgins PG. An outbreak of adenovirus keratoconjunctivitis in Bristol. Br J Ophthalmol. 1979;63(9):621-626.

17. Zhou X, Robinson CM, Rajaiya J, et al. Analysis of human adenovirus type 19 associated with epidemic keratoconjunctivitis and its reclassification as adenovirus type 64. Invest Ophthalmol Vis Sci. 2012; 53(6):2804-2811.

18. Aoki K, Kato M, Ohtsuka H, Ishii K, Nakazono N, Sawada H. Clinical and aetiological study of adenoviral conjunctivitis, with special reference to adenovirus types 4 and 19 infections. Br J Ophthalmol. 1982; 66(12):776-780

19. Darougar S, Grey RH, Thaker U, McSwiggan DA. Clinical and epidemiological features of adenovirus keratoconjunctivitis in London. Br J Ophthalmol. 1983;67(1):1-7.

20. Huang G, Yao W, Yu W, et al. Outbreak of epidemic keratoconjunctivitis caused by human adenovirus type 56, China, 2012. PLoS One. 2014;9(10):e110781.

21. Laibson PR, Dhiri S, Oconer J, Ortolan G. Corneal infiltrates in epidemic keratoconjunctivitis. Response to double-blind corticosteroid therapy. Arch Ophthalmol. 1970;84(1):36-40.

22. Zhang X, Li Q, Xiang M, et al. Bulbar conjunctival thickness measurements with optical coherence tomography in healthy Chinese subjects. Invest Ophthalmol Vis Sci. 2013;54(7):4705-4709.

23. Ghanaiem H, Averbuch D, Koplewitz BZ, et al. An outbreak of adenovirus type 7 in a residential facility for severely disabled children. Pediatr Infect Dis J. 2011;30(11):948-952.

24. James L, Vernon MO, Jones RC, et al. Outbreak of human adenovirus type 3 infection in a pediatric long-term care facility-Illinois, 2005. Clin Infect Dis. 2007;45(4):416-420.

25. Calkavur S, Olukman O, Ozturk AT, et al. Epidemic adenoviral keratoconjunctivitis possibly related to ophthalmological procedures in a neonatal intensive care unit: lessons from an outbreak. Ophthalmic Epidemiol. 2012;19(6):371-379.

26. Koc J, Wigand R, Weil M. The efficiency of various laboratory methods for the diagnosis of adenovirus conjunctivitis. Zentralbl Bakteriol Mikrobiol Hyg A. 1987;263(4):607-615.

27. Sarbay H, Polat A, Mete E, Balci YI, Akin M. Pertussis-like syndrome associated with adenovirus presenting with hyperleukocytosis: Case report. North Clin Istanb. 2016;3(2):140-142.
Clinical Ophthalmology

\section{Publish your work in this journal}

Clinical Ophthalmology is an international, peer-reviewed journal covering all subspecialties within ophthalmology. Key topics include: Optometry; Visual science; Pharmacology and drug therapy in eye diseases; Basic Sciences; Primary and Secondary eye care; Patient Safety and Quality of Care Improvements. This journal is indexed on Submit your manuscript here: http://www.dovepress.com/clinical-ophthalmology-journal

\section{Dovepress}

PubMed Central and CAS, and is the official journal of The Society of Clinical Ophthalmology (SCO). The manuscript management system is completely online and includes a very quick and fair peer-review system, which is all easy to use. Visit http://www.dovepress.com/ testimonials.php to read real quotes from published authors. 